\title{
Passive Bilateral Teleoperation Framework for Assisted Robotic Tasks
}

\author{
Emmanuel Nuño and Luis Basañez \\ Institute of Industrial and Control Engineering (IOC) \\ Technical University of Catalonia (UPC) \\ Barcelona 08028, Spain. \\ \{emmanuel.nuno, luis.basanez\}@upc.edu
}

\author{
Romeo Ortega \\ Laboratoire des Signaux et Systèmes \\ CNRS-SUPÉLEC \\ Gif-sur-Yvette 91192, France. \\ ortega@lss.supelec.fr
}

\begin{abstract}
This paper addresses the problem of stable bilateral teleoperation, through a time-delayed communication channel, for a $n$-DOF system without position drift. The classical wave variables-based scheme encodes velocity and force whilst the proposed scheme encodes position and integral of force. The resulting passive communication scheme is used in an extended teleoperation framework, in which the human operator can define geometrical motion restrictions in order to increase his awareness and his feeling of immersion, hence improving his ability to perform complex tasks. The framework also provides the operator with video feedback and 3D rendering of the remote scene.
\end{abstract}

\section{INTRODUCTION}

In bilateral teleoperation, the master and the slave are connected with a communication channel that often involves large distances or impose limited data transfer between the local and the remote sites. Such situations can result in substantial delays between the time a command is introduced by the operator and the time the command is executed by the remote robot. This time-delay affects the overall stability of the system [1]. Passivity and scattering theories helped Anderson and Spong [2] to introduce the wave variables for teleoperation systems, and, following their formalism, Niemeyer and Slotine [3] presented a more intuitive, wave variables approach. Since then, the use of wave variables has been widely extended [4], [5], [6]. Alise et al. [7] provided additional tuning flexibility for the $n$-DOF wave variable method, and their generalization has been studied for the teleoperation scheme proposed here. Recently other passivity based controllers have been developed: Lee and $\mathrm{Li}$ [8] formulate the passive decomposition regulating the masterslave position coordination; Ryu et al. [9] apply the passivity observer and passivity controller stabilization method using a time-variable energy threshold for the observer; Mahvash and Hayward [10] use the passivity theory in the creation of virtual environments where the slave and the communication channel are replaced by a force-reflecting simulated environment; Lee and Spong [11] propose, in a rather intriguing note, a teleoperation control scheme based on a PD control law which enforces position coordination.

The traditional wave variable method encodes velocity and force and this codification is transmitted to the slave. This method solves the stability problem but position drift arises. Chopra et al. [12] propose a wave based control scheme that uses an additional position control on both the master and the slave. Niemeyer and Slotine [13] study the behavior of the system when transmitting the wave integral, and propose that the communications should transmit both wave and integrated wave signals. In order to avoid the position drift the teleoperation scheme proposed here, reported by Ortega et al. [14], makes use of wave variables that codify position and integral of force. This communication scheme is applied to a wider robot teleoperation framework under development. This teleoperation framework allows the operator to define motion restrictions which depend on the task to be performed. On the master side, the deviation from the restriction generates an attractive force to the restriction subspace, providing the operator with an intuitive interface to ensure movements inside this subspace. Motion restrictions can be easily defined and modified by the operator and can highly improve the task performance besides the sensation of immersion. The operator is also provided with a 3D viewer of the remote scene. Combining this position drift free scheme with motion restrictions is one of the main contributions of this paper.

In order to assist the operator while performing different tasks some approaches have already been developed. Turro et al. [15] have implemented three types of constraints for the operator movements: movement constraint along a line, virtual obstacle avoidance using a potential field force and geometric cube constraint in order to limit the robot workspace. However, this approach needs to be reprogrammed when a new restriction must be introduced and moreover this teleoperation scheme does not guarantee stability with time-delay. Casavola and Sorbara [16] present a constrained teleoperation system that has been developed using predictive control techniques. The constraints act in the nominal path of the robot end-effector, but motion guidance is not implemented on the master side, and the operator does not have the corresponding feedforward force.

This paper is organized as follows: Section II outlines the proposed teleoperation framework with its subsystems; Section III presents a description of the traditional wave variables, and Section IV depicts the proposed position drift free scheme with some simulations in Section IV-B; the experimental testbed with a experiment of motion along a line restriction over a rail is depicted in Section V. Finally in Section VI some conclusions and future work are proposed. 


\section{TELEOPERATION FRAMEWORK}

The proposed teleoperation framework is shown in figure 1. It has three subsystems which are well differentiated: the Local Command Center, where information concerning to geometrical restrictions and guidance is computed; the Communication Channel, that manages all information flow; and the Remote Robotic Cell, where the desired task is performed.

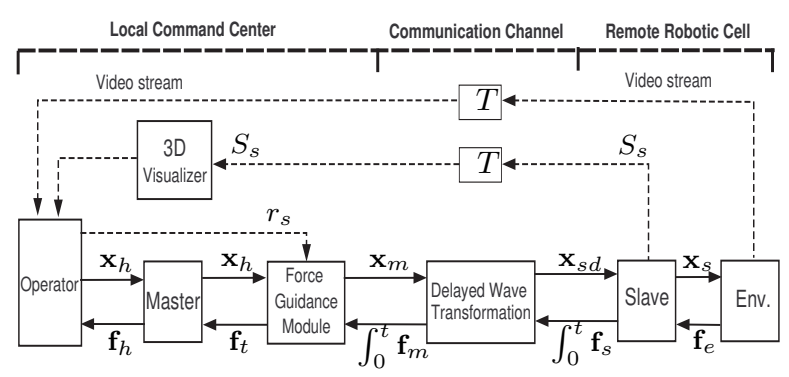

Fig. 1. Teleoperation framework scheme

\section{A. Local Command Center}

This subsystem handles the forces that have to be fed back to the operator as well as the integration of position information with the motion restrictions. In addition to the main control loop, a video stream provides video feedback from cameras located at the Remote Robotic Cell, whose zoom and orientation can be remotely actuated. A 3D visualizer provides the operator with information concerning the position and orientation of the robot end-effector, this visualizer is aimed to reduce the traffic in the network produced by the video feedback which is a high-bandwidth consumer. The visualizer with a $3 \mathrm{D}$ scene is depicted in figure 2.

The Force Guidance Module fulfills two different functions: 1) The definition by the operator of a motion restriction $r_{s} ; 2$ ) The computation of the restriction force $\mathbf{f}_{r}$ that must be exerted to maintain the position of the end-effector inside the currently selected motion restriction $r_{s}$, as well as of the viscous force $\mathbf{f}_{v}$ that prevents the velocity of the end-effector from becoming too large for the robot to follow.

The restriction force $\mathbf{f}_{r}$ and viscous force $\mathbf{f}_{v}$ are combined with the master force $\mathbf{f}_{m}$ of the wave variable method to generate the total force $\mathbf{f}_{t}$, which is fed to the operator via a haptic device. The total force is $\mathbf{f}_{t}=\mathbf{f}_{m}+\mathbf{f}_{r}+\mathbf{f}_{v}$.

-Master force $\mathbf{f}_{m}$. Depending on the communication control scheme used, the master force varies. The next section shows the appropriate master force for the presented scheme.

-Restriction force $\mathbf{f}_{r}$. Is an attractive force that tends to fix the haptic position to the restriction subspace. It is modeled as a spring-damping system. At instant $k, \mathbf{f}_{r}$ is given by $\mathbf{f}_{r}=K_{p} \mathbf{e}+\mathbf{d}$ where $\mathbf{d}$ is the corresponding damping part of the controller and it is given by $\mathbf{d}=K_{d}\left(\mathbf{e}_{k}-\mathbf{e}_{k-1}\right)$; $\mathbf{e}$ is the position deviation: $\mathbf{e}=\mathbf{x}_{r}-\mathbf{x}_{h d} ; \mathbf{x}_{r}$ is the reference point that lies on the restriction subspace and $\mathbf{x}_{h d}$ is the position introduced by the human operator. The value of $\mathbf{f}_{r}$ will be

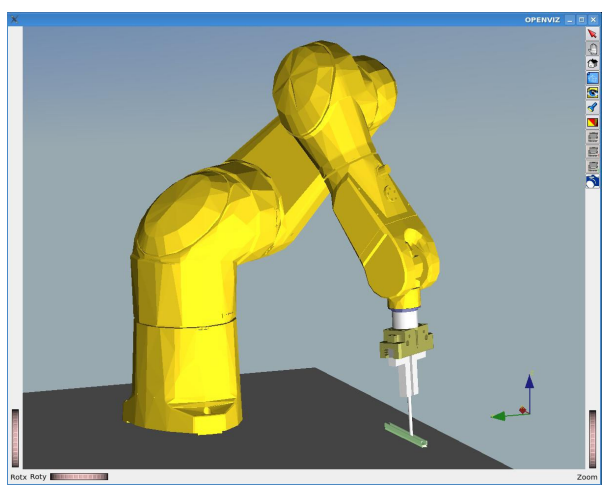

Fig. 2. 3D rendering of the remote scene

zero if no restriction is set. $K_{p}$ and $K_{d}$ are chosen to set the stiffness and damping of the restriction.

-Viscous force $\mathbf{f}_{v}$. When the velocity of the master is too high, the slave may not be able to follow the commands. In order to deal with this problem an additional restriction has been implemented: above a certain velocity value, $\dot{\mathbf{x}}_{M}$, which depends on the maximum velocity achievable by the slave, the motion is restricted using a function of the master velocity $\dot{\mathbf{x}}_{m}$. The resulting force of this effect for $\dot{\mathbf{x}}_{m}>\dot{\mathbf{x}}_{M}$ is given by $\mathbf{f}_{v}=K_{v} \dot{\mathbf{x}}_{m}$, where $K_{v}$ is a gain that fits the needs of restrict velocity.

The 3D visualizer receives state signals $\left(S_{s}\right)$ from the slave that give the operator information of the remote environment. This visualizer contents a virtual 3D model of the remote scene which components are dynamic. For example, if an obstacle changes its position in the environment it also changes in the visualizer. This is made just by sending the new position of the obstacle, hence, optimizing the usage of bandwidth.

It is important to stress the difference between the three components of the total force. Whilst the master force represents a feedback signal -the reaction arising from the interaction of the robot with its environment- the restriction and viscous forces represent feedforward signals in the sense that they respond to known inputs -the deviations from the restriction subspace and the velocity limitation, respectivelywithout the need of any information from the workcell. The motion restriction $r_{s}$ is updated at a much lower frequency than the other signals.

\section{B. Remote Robotic Cell}

Here resides the slave robot controller which depending on the task, guides the robot strictly inside the restriction subspace $\left(\mathbf{x}_{r}\right)$ or following the human position $\left(\mathbf{x}_{h d}\right)$ with a deviation (e) allowed by the stiffness and damping from the Force Guidance Module. Figure 3 depicts the implemented geometric restrictions.

\section{Communication Channel}

The communication channel in this framework is implemented following the scheme proposed in chapter IV of position drift free teleoperation. It internally encodes position 


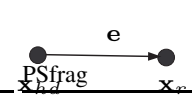

a) Point

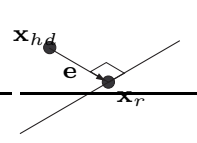

b) Line

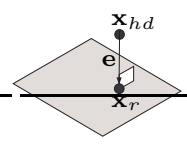

c) Plane

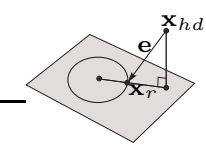

d) Circle
Fig. 3. Geometrical restrictions

and integral of force into wave variables, transmitted in each direction, in order to render passive the time-delayed communication channel. Other important function of this subsystem is to manage the communication needs for the video stream and the 3D visualizer (the video stream consumes lot of bandwidth the 3D visualizer does not). It has been implemented using sockets UDP with the new IPv6 protocol, providing higher QoS (Quality of Service) to the control information and 3D visualizer data flow than the video stream.

\section{The Traditional Wave Variable Method}

In the wave variable approach, velocity $\dot{x}$ and force $f$ are encoded with an appropriate transformation and only the resulting wave variable is transmitted. The master $(m)$ and slave $(s)$ robots are passive mechanical systems from force to velocity. The equations governing a constant time-delay communication channel are

$$
\begin{aligned}
& \mathbf{u}_{s}(t)=\mathbf{u}_{m}(t-\tau) \\
& \mathbf{v}_{m}(t)=\mathbf{v}_{s}(t-\tau)
\end{aligned}
$$

where $\tau$ is the communication delay. The wave transformation for a one DOF system is

$$
\begin{aligned}
\mathbf{u}_{m}(t) & =\frac{1}{\sqrt{2 b}}\left(\mathbf{f}_{m}+b \dot{\mathbf{x}}_{m}\right) & \mathbf{v}_{m}(t) & =\frac{1}{\sqrt{2 b}}\left(\mathbf{f}_{m}-b \dot{\mathbf{x}}_{m}\right) \\
\mathbf{u}_{s}(t) & =\frac{1}{\sqrt{2 b}}\left(\mathbf{f}_{s}+b \dot{\mathbf{x}}_{s d}\right) & \mathbf{v}_{s}(t) & =\frac{1}{\sqrt{2 b}}\left(\mathbf{f}_{s}-b \dot{\mathbf{x}}_{s d}\right)
\end{aligned}
$$

where $b>0$ is an arbitrary damping parameter. How passivity is established in this approach is explained using the scattering operator norm from the hybrid matrix of the teleoperation system. Let us define $\mathbf{H}(s)$ the hybrid matrix in the Laplace domain as

$$
\left[\begin{array}{c}
\mathbf{F}_{m} \\
-s \mathbf{X}_{s d}
\end{array}\right]=\mathbf{H}(s)\left[\begin{array}{c}
s \mathbf{X}_{m} \\
\mathbf{F}_{s}
\end{array}\right]
$$

The corresponding hybrid matrix for the wave transformation is equal to

$$
\mathbf{H}(s)=\left[\begin{array}{cc}
\frac{b\left(1-e^{-2 s \tau}\right)}{1+e^{-2 s \tau}} & \frac{1+b}{e^{s \tau}+e^{-s \tau}} \\
\frac{-2}{e^{s \tau}+e^{-s \tau}} & \frac{1-e^{-2 s \tau}}{1+e^{-2 s \tau}}
\end{array}\right]
$$

The scattering operator $\mathbf{S}(s)$ is related to the hybrid matrix by the expression

$$
\mathbf{S}(s)=[\mathbf{H}(s)-\mathbf{I}][\mathbf{H}(s)+\mathbf{I}]^{-1}
$$

Anderson and Spong [2] state that an LTI $n$-port system is passive if and only if the norm of its scattering operator is less than or equal to one $\|\mathbf{S}(s)\| \leq 1$. Finding the scattering operator and calculating its norm, it can be seen that the wave transformation fulfils the norm condition, hence it is passive independently of the magnitude of the delay $\tau$ if zero initial conditions are assumed. The wave energy is thus temporarily stored whilst in transit, making the communication channel passive. Although the strictly positive parameter $b$ can be chosen arbitrarily, it defines a characteristic impedance associated with the wave variables and directly affects the system behavior [3]. However, although this wave variable method renders passive the communication channel it incurs in position drift due to the velocity control in the slave. This position error is function of the initial conditions $x_{m}(0), x_{s d}(0), x_{s}(0)$ and the integral of $\dot{x}_{s d}$. The additional tuning flexibility provided by Alise et al. [7] for the wave variable method makes use of the transformation

$$
\begin{aligned}
\mathbf{u}_{m} & =\mathbf{A}_{w} \dot{\mathbf{x}}_{m}+\mathbf{B}_{w} \mathbf{f}_{m} & \mathbf{v}_{m} & =\mathbf{C}_{w} \dot{\mathbf{x}}_{m}-\mathbf{D}_{w} \mathbf{f}_{m} \\
\mathbf{u}_{s} & =\mathbf{A}_{w} \dot{\mathbf{x}}_{s d}+\mathbf{B}_{w} \mathbf{f}_{s} & \mathbf{v}_{s} & =\mathbf{C}_{w} \dot{\mathbf{x}}_{s d}-\mathbf{D}_{w} \mathbf{f}_{s}
\end{aligned}
$$

which also renders passive the communication channel by choosing the appropriate combination of matrices, which should be: $\mathbf{A}_{w} \in \Re^{n x n}$ nonsingular for $n$ DOF, $\mathbf{B}_{w}=$ $\frac{1}{2} \mathbf{A}_{w}^{-T}, \mathbf{C}_{w}=\mathbf{Q} \mathbf{A}_{w}$ being $\mathbf{Q} \in \Re^{n x n}$ orthogonal and finally $\mathbf{D}_{w}=\frac{1}{2} \mathbf{Q} \mathbf{A}_{w}^{-T}$. If $\mathbf{A}_{w}=\sqrt{\frac{b}{2}} \mathbf{I}$ and $\mathbf{Q}=\mathbf{I}$ the above equations coincide with eq. 2 for one DOF.

\section{Proposed Position Drift Free Teleoperation}

\section{A. Formulation}

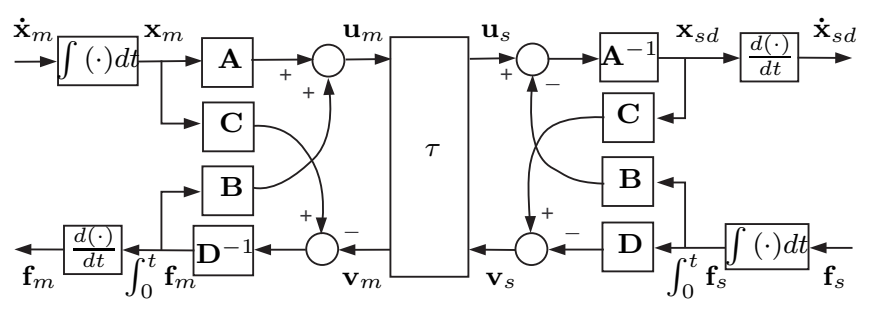

Fig. 4. Position drift free scheme based on wave variables

In order to avoid position drift, it is possible to adopt the alternative scattering transformation given by (Figure 4)

$$
\begin{aligned}
\mathbf{u}_{m} & =\mathbf{A}_{w} \mathbf{x}_{m}+\mathbf{B}_{w} \int_{0}^{t} \mathbf{f}_{m}(t) d t & \mathbf{v}_{m} & =\mathbf{C}_{w} \mathbf{x}_{m}-\mathbf{D}_{w} \int_{0}^{t} \mathbf{f}_{m}(t) d t \\
\mathbf{u}_{s} & =\mathbf{A}_{w} \mathbf{x}_{s d}+\mathbf{B}_{w} \int_{0}^{t} \mathbf{f}_{s}(t) d t & \mathbf{v}_{s} & =\mathbf{C}_{w} \mathbf{x}_{s d}-\mathbf{D}_{w} \int_{0}^{t} \mathbf{f}_{s}(t) d t
\end{aligned}
$$

Comparing (2) and (3) we observe the introduction of integral actions. This codification of position $\mathrm{x}$ and integral of force $\int_{0}^{t} \mathbf{f} d t$ renders passive the communication channel and moreover enables the teleoperation system to have position tracking, resulting on a position drift free scheme. In order to prove that this configuration retains the passivity of the overall system, let us define the conditions for the matrices [4]. $\mathbf{A}_{w}$ must be nonsingular and the following equations must be fulfilled

$$
\begin{array}{cc}
\mathbf{B}_{w}=\frac{1}{2} \mathbf{A}_{w}^{-T} & \mathbf{C}_{w}^{T} \mathbf{C}_{w}=\mathbf{A}_{w}^{T} \mathbf{A}_{w} \\
\mathbf{D}_{w}^{T} \mathbf{D}_{w}=\mathbf{B}_{w}^{T} \mathbf{B}_{w} & \mathbf{A}_{w}^{T} \mathbf{B}_{w}+\mathbf{C}_{w}^{T} \mathbf{D}_{w}=\mathbf{I}
\end{array}
$$

Let the supply rate $P_{\text {in }}$ be defined as the scalar product between the input $\mathbf{x}$ and the output $\mathbf{y}$. This system is said to be passive if and only if

$$
\int_{0}^{t} P_{\text {in }} d \tau=\int_{0}^{t} \mathbf{x}^{T} \mathbf{y} d \tau=E_{\text {store }}(t)-E_{\text {store }}(0) \geq 0
$$


where $E_{\text {store }}$ is the storage function. Then the power flowing in the communication channel from 0 to $t$ will be

$$
P_{i n}(t)=\mathbf{x}_{m}^{T}(t) \int_{0}^{t} \mathbf{f}_{m}(t) d t-\mathbf{x}_{s d}^{T}(t) \int_{0}^{t} \mathbf{f}_{s}(t) d t
$$

Substituting (1) and (3) into (5) and taking $\mathbf{C}_{w}=\mathbf{A}_{w}$ and $\mathbf{D}_{w}=\mathbf{B}_{w}$, which fulfil the conditions of (4), it is clearly seen that the storage function increase while the transmission between the master and the slave takes place, and it is given by

$$
\begin{aligned}
E(t)= & \frac{1}{4} \int_{0}^{t}\left(\mathbf{u}_{m}^{T} \mathbf{A}_{w}^{-T} \mathbf{B}_{w}^{-1} \mathbf{u}_{m}-\mathbf{v}_{m}^{T} \mathbf{A}_{w}^{-T} \mathbf{B}_{w}^{-1} \mathbf{v}_{m}\right) d t \\
& -\frac{1}{4} \int_{0}^{t}\left(\mathbf{u}_{s}^{T} \mathbf{A}_{w}^{-T} \mathbf{B}_{w}^{-1} \mathbf{u}_{s}+\mathbf{v}_{s}^{T} \mathbf{A}_{w}^{-T} \mathbf{B}_{w}^{-1} \mathbf{v}_{s}\right) d t
\end{aligned}
$$

Using (1) the energy function becomes

$$
E(t)=\underbrace{\frac{1}{4} \int_{t-\tau}^{t} \mathbf{u}_{m}^{T} \mathbf{A}_{w}^{-T} \mathbf{B}_{w}^{-1} \mathbf{u}_{m} d t}_{E_{m}(t)}+\underbrace{\frac{1}{4} \int_{t-\tau}^{t} \mathbf{v}_{s}^{T} \mathbf{A}_{w}^{-T} \mathbf{B}_{w}^{-1} \mathbf{v}_{s} d t}_{E_{s}(t)}
$$

by construction $\mathbf{A}_{w}$ and $\mathbf{B}_{w}$ are diagonal matrices whose elements are positive and then its multiplication is a positive definite matrix, which its inverse is also positive definite. Hence $E_{m}(t), E_{s}(t) \geq 0 \forall t$, and therefore the system is passive independent of the magnitude of the delay $\tau$. Position drift is eliminated although the initial position of the master and the slave may differ.

When $\mathbf{f}_{s}>0$ means that the slave has reached an obstacle, and when it leaves this force becomes null. The integral of this force gives rise to an undesired side effect which is known as integrator windup, hence becomes necessary the creation of an anti wind-up compensator scheme, this approach makes use of the scheme proposed by Bohn and Atherton in [17] (Fig. 5).

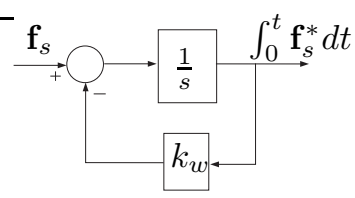

Fig. 5. Wind-up compensator with $k_{w} \in \Re^{+}$

The use of this compensator does not affect the passivity of the method, the new integral becomes the convolution

$$
\int_{0}^{t} \mathbf{f}_{s}^{*} d t=\int_{0}^{t} \mathbf{f}_{s}(\varphi) e^{-k_{w}(t-\varphi)} d \varphi
$$

following that $0<e^{-k_{w}(t-\varphi)} \leq 1 ; \forall t<\infty$, with $k_{w}$ being a positive constant, the convolution is always less than or equal to the integral of force; in other words

$$
\int_{0}^{t} \mathbf{f}_{s}(\varphi) e^{-k_{w}(t-\varphi)} d \varphi \leq \int_{0}^{t} \mathbf{f}_{s}(\varphi) d \varphi
$$

and the power input equation (5) for the system turns into $P_{i n}(t)=\mathbf{x}_{m}^{T}(t) \int_{0}^{t} \mathbf{f}_{m}(t) d t-\mathbf{x}_{s d}^{T}(t) \int_{0}^{t} \mathbf{f}_{s}(\varphi) e^{-k_{w}(t-\varphi)} d \varphi$ the total energy on the communication channel $E(t)$ remains positive, which means that the energy in the outgoing wave is greater than or equal to the energy in the returning wave, hence the system continues being passive.

\section{B. Simulation}

In this section a simulation of the aforementioned teleoperation scheme is presented. The master and the slave are modeled as a pair of 2 DOF serial links. The corresponding nonlinear dynamics follow the next equations:

$$
\begin{gathered}
\mathbf{M}_{m}\left(\mathbf{x}_{m}\right) \ddot{\mathbf{x}}_{m}+\mathbf{V}\left(\mathbf{x}_{m}, \dot{\mathbf{x}}_{m}\right)+\mathbf{G}\left(\mathbf{x}_{m}\right)=\mathbf{f}_{m}-\mathbf{f}_{h} \\
\mathbf{M}_{s}\left(\mathbf{x}_{s}\right) \ddot{\mathbf{x}}_{s}+\mathbf{V}\left(\mathbf{x}_{s}, \dot{\mathbf{x}}_{s}\right)+\mathbf{G}\left(\mathbf{x}_{s}\right)=\mathbf{f}_{s}-\mathbf{f}_{e}
\end{gathered}
$$

where $\mathbf{M}_{i} \in \Re^{2 \times 2}$ ( $i=1$ for the master and $i=2$ for the slave) is the inertia matrix given by

$$
\mathbf{M}_{i}\left(\mathbf{x}_{i}\right)=\left[\begin{array}{cc}
\alpha_{i}+2 \beta_{i} \cos \left(x_{2_{i}}\right) & \delta_{i}+\beta_{i} \cos \left(x_{2_{i}}\right) \\
\delta_{i}+\beta_{i} \cos \left(x_{2_{i}}\right) & \delta_{i}
\end{array}\right]
$$

$x_{k_{i}}, k \in\{1,2\}$ is the articular position of each link, $\alpha_{i}=$ $l_{2_{i}}^{2} m_{2_{i}}+l_{1_{i}}^{2}\left(m_{1_{i}}+m_{2_{i}}\right), \beta_{i}=l_{1_{i}} l_{2_{i}} m_{2_{i}}$ and $\delta_{i}=l_{2_{i}}^{2} m_{2_{i}}$. The length of the both links $l_{1_{i}}$ and $l_{2_{i}}$ are $0.38 \mathrm{~m}$. The mass of each link correspond to $m_{1_{m}}=3.9473 \mathrm{~kg}, m_{2_{m}}=$ $0.6232 \mathrm{~kg}, m_{1_{s}}=3.2409 \mathrm{~kg}$ and $m_{2_{s}}=0.3185 \mathrm{~kg}$. These values are the same of those used by Lee and Spong in [11]. Coriolis and centrifugal forces are modeled as the vector $\mathbf{V}_{i} \in \Re^{2 \times 1}$ which is

$$
\mathbf{V}_{i}\left(\mathbf{x}_{i}, \dot{\mathbf{x}}_{i}\right)=\left[\begin{array}{c}
-\beta_{i} \sin \left(x_{2_{i}}\right) \dot{x}_{2_{i}}^{2}-\beta_{i} \sin \left(x_{2_{i}}\right) \dot{x}_{1_{i}} \dot{x}_{2_{i}} \\
\beta_{i} \sin \left(x_{2_{i}}\right) \dot{x}_{1_{i}}^{2}
\end{array}\right]
$$

$\dot{x}_{1_{i}}$ and $\dot{x}_{2_{i}}$ are the respective revolute velocities of the two links. The gravity effect $\left(\mathbf{G}_{i} \in \Re^{2 \times 1}\right)$ in each link is represented as

$\mathbf{G}_{i}\left(\mathbf{x}_{i}\right)=\left[\begin{array}{c}\frac{1}{l_{2_{i}}} g \delta_{i} \cos \left(x_{1_{i}}+x_{2_{i}}\right)+\frac{1}{l_{1_{i}}}\left(\alpha_{i}-\delta_{i}\right) \cos \left(x_{1_{i}}\right) \\ \frac{1}{l_{2_{i}}} g \delta_{i} \cos \left(x_{1_{i}}+x_{2_{i}}\right)\end{array}\right]$

$\mathbf{f}_{h}$ and $\mathbf{f}_{e}$ are the operator and environmental forces. The master force $\mathbf{f}_{m}$ is given by

$$
\mathbf{f}_{m}(t)=\mathbf{D}_{w}^{-1} \frac{d}{d t}\left(\mathbf{C}_{w} \mathbf{x}_{m}(t)-\mathbf{v}_{m}(t)\right)
$$

and the slave force $\mathbf{f}_{s}$ is generated by a PD controller

$$
\mathbf{f}_{\mathrm{s}}=\mathbf{K}_{s}\left(\mathbf{x}_{s d}-\mathbf{x}_{s}\right)+\mathbf{B}_{s}\left(\dot{\mathbf{x}}_{s d}-\dot{\mathbf{x}}_{s}\right)
$$

$\mathbf{K}_{s}=4 \mathbf{I}_{2 \times 2} \frac{N}{m}$ and $\mathbf{B}_{s}=7 \mathbf{I}_{2 \times 2} \frac{N s}{m}$ are gain matrices, the transfer function of the PD controller is proper with a derivative filter constant equals to $\alpha B_{s_{1,1}} / K_{s_{1,1}}$ being $\alpha=0.1$, the desired position $\mathbf{x}_{s d}$ is calculated as

$$
\mathbf{x}_{s d}=\mathbf{A}_{w}^{-1}\left(\mathbf{u}_{s}-\mathbf{B}_{w} \int_{0}^{t} \mathbf{f}_{s}(\varphi) e^{-k_{w}(t-\varphi)} d \varphi\right)
$$

$\mathbf{f}_{e}$ matches a spring-damper system with gains $10000 \frac{\mathrm{N}}{\mathrm{m}}$ and $1 \frac{N s}{m}$ respectively. Matrix $\mathbf{A}_{w}$ is equal to $(b / 2)^{1 / 2} \mathbf{I}_{2 \times 2}$, the other matrices were calculated using eq (4). The timedelay $\tau$ in both, the forward and return paths, is fixed to $2 s$. The constant $k_{w}$ for the anti-windup is equals to 0.05 . The simulation has been carried out using MatLab SimuLink ${ }^{\mathrm{TM}}$. 

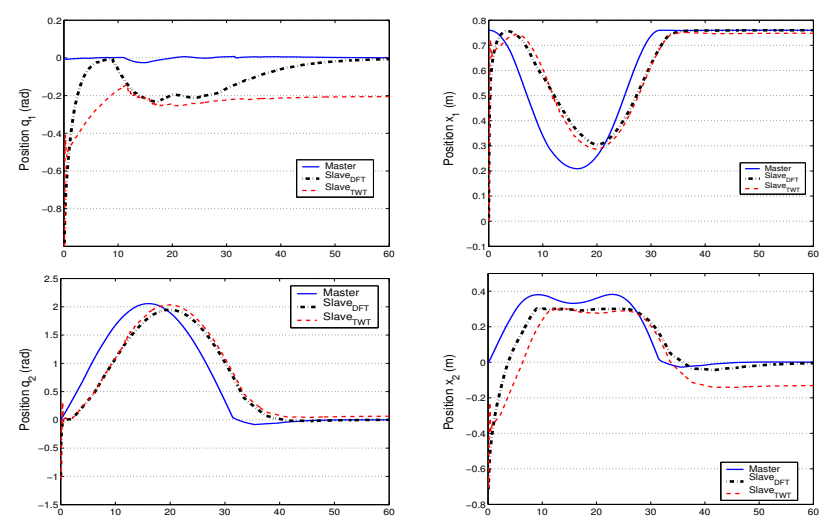

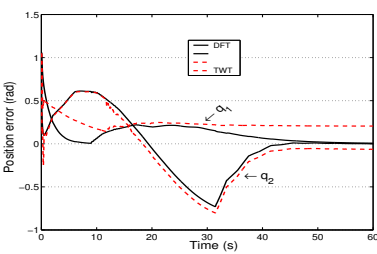

a) Articular space

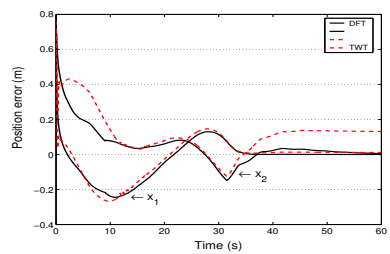

b) Cartesian space
Fig. 6. Position and error for the master and the slave with $\tau=2 \mathrm{~s}$. DFT (Drift Free Teleoperation), TWT (Traditional Wave Teleoperation).

In order to evaluate the stability of the proposed scheme a stiff wall $\left(10000 \frac{\mathrm{N}}{\mathrm{m}}\right)$ in the environment at $x_{2}=0.3 \mathrm{~m}$ has been included. The initial conditions for the master and the slave differ one from the other, $\mathbf{x}_{m}(0)=[0,0]^{T}$ and $\mathbf{x}_{s}(0)=[-1 / 3 \pi,-1 / 3 \pi]^{T}$. Both, the position drift free and the traditional wave scheme have been simulated with the same circumstances. Figure 6.a presents the results of the simulation for the articular values and figure $6 . b$ for the cartesian space. It can be seen for the position drift free scheme that the slave follows the desired position and although the time-delay $(\tau=2 s)$ is quite substantial the whole system is stable. At $x_{2}=0.3 m$ the slave reaches the obstacle around $10 \mathrm{~s}$ and reflects the force interaction to the master. Although the initial position of the slave is not the same as in the master it converges to the desired position. The plots of the traditional wave teleoperation exhibit a position drift arising from the interaction with the environment.

\section{EXPERIMENTAL TEST-BED}

Figure 7 shows the experimental testbed that mainly consists of a TX-90 Stäubli robot with a CS8-C Stäubli controller and a JR3 force-torque sensor, a PHANToM $1.5^{\mathrm{TM}}$ 6DOF haptic device from Sensable Technologies, and two CANON VC-C5 video cameras with an AXIS 2400 video server which provides a 10-20 fps motion JPEG video stream. On the software side, the interaction with the haptic device makes use of the Sensable Technologies' $\mathrm{GHoST}^{\mathrm{TM}}$ libraries. The haptic's control loop runs at $1 \mathrm{kHz}$, and forces must be calculated within the millisecond time window. All software is written in $\mathrm{C}++$ using sockets and POSIX threads. The Graphic User Interface has been developed with Trolltech's QT library. The 3D visualizer is implemented using the COIN 3D libraries with the Flex++ and Bison parsers.

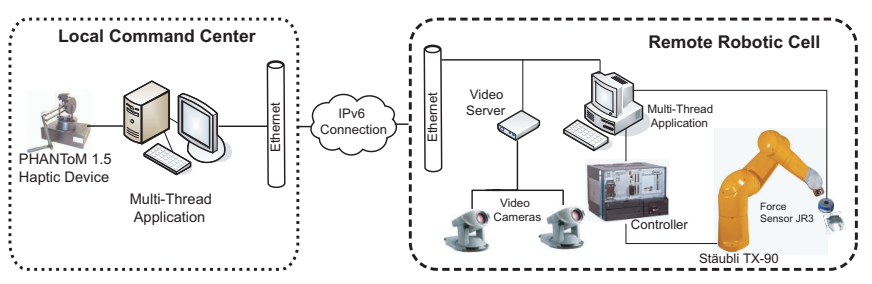

Fig. 7. Physical system architecture

\section{A. Test-Motion with a Line Restriction over a Rail-}

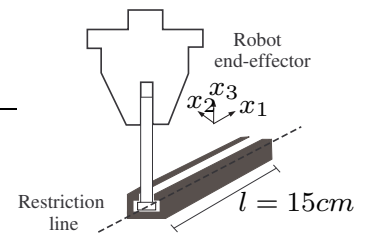

Fig. 8. Line restriction over a rail

In order to validate the proposed approach an experimental test was remotely performed using the teleoperation framework with the passive position drift free scheme. It consists on moving the robot end-effector along a rail with a line restriction.

The proposed test has the following characteristics:

a) The motion of the robot end-effector is restricted to a line in the $x_{1}$ axis as shown in figure 8 .

b) The forces coming from the remote robotic cell $\mathbf{f}_{m}$ provide information about the interaction of the end effector with the environment.

c) The position commands $\mathbf{x}_{m}$ correspond to the master position reflected on the restricted line, namely $\mathbf{x}_{r}$.

d) Packets have been transmitted using TCP/IPv6 sockets with the scheme of a classical client-server application, providing higher IPv6 QoS to control commands than the video transmission.

e) In order to illustrate the test the orientation and the torques have been omitted. Although the $6 \mathrm{DOF}$ of the robot have been controlled.

Figure 9.a plots the time evolution of positions and forces along the $x_{1}, x_{2}$ and $x_{3}$ axis. The force figures show two of the three components of the total force $\mathbf{f}_{t}$ : the restriction force $\mathbf{f}_{r}$ and the master force $\mathbf{f}_{m}$ (due to the low velocity of the experiment the viscous force, $\mathbf{f}_{v}$, is negligible). The line restriction is along the $x_{1}$ axis.

The graphics of the figure 9.a describe the motion of the robot along the restricted subspace $\left(\mathbf{x}_{r}\right)$. The graphics have three zones separated by dashed vertical lines: zone A corresponds to free space without motion restriction; zone $\mathrm{B}$ the end effector moves along the rail restricted to a line; and in zone $\mathrm{C}$ the end effector moves in free space. In these graphics it can be seen that when the restriction is set (at around 10s) position in $x_{2}$ and $x_{3}$ axis goes to the origin, and motion only takes place in the $x_{1}$ direction. Since the task has been performed at low speed, the viscous force $\left(\mathbf{f}_{v}\right)$ 

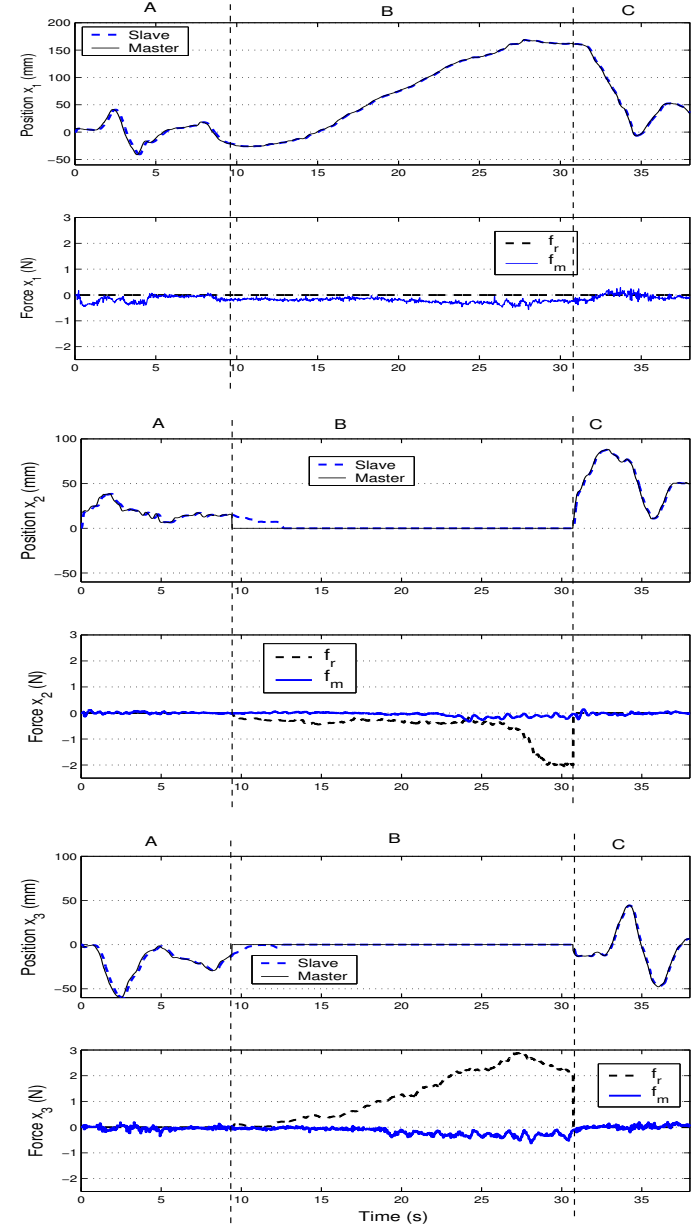

a) Positions and forces
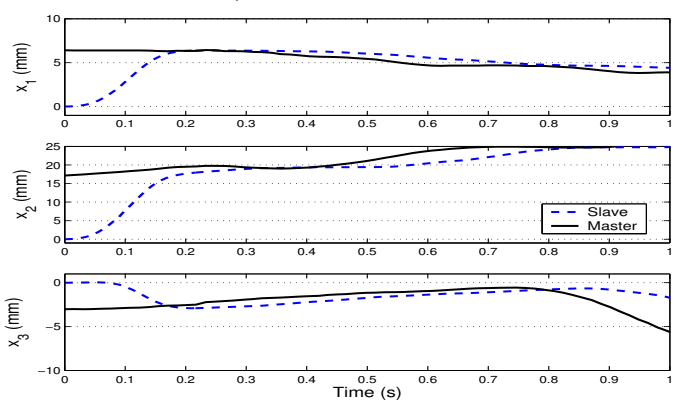

b) Initial position offset

Fig. 9. Experimental results

does not have a significant contribution to the total force, and the restriction force is dominated by its spring component.

Figure 9.b depicts the initial positions of both, the master and the slave, despite its difference the position error converges to zero with time, shown that the position drift has been eliminated.

\section{CONCLuding Remarks}

The presented teleoperation framework makes use of a passive communication channel that codifies position and integral of force instead of the velocity and force of the traditional wave teleoperation, resulting on a position drift free scheme. The framework can lower the burden on the operator while remotely executing a task, since the operator is provided, in addition to the force feedback, with a feed forward force that guides its motion according to some geometric constraints. The presented approach has been validated with some simulations comparing the traditional and the proposed teleoperation frameworks and also with a experimental test of motion with a line restriction over a rail. Due to space limitations, the experiments only show the results of a line restriction, however, the other geometric restrictions (section II-B) have been tested giving similar results.

\section{ACKNOWLEDGMENTS}

The authors would like to gratefully acknowledge the support of Leopold Palomo, Adolfo Rodríguez and Patrick Grosch with this project. This work has been partially supported by the spanish CICYT projects: DPI2005-00112 and DPI2004-03104, the FPI program with reference BES-200613393 , and also by the mexican CONACyT grant- 169003 .

\section{REFERENCES}

[1] T.B. Sheridan. Space teleoperation through time delay: review and prognosis. IEEE Trans. Robot. Autom., 9(5):592-606, Oct. 1993.

[2] R.J. Anderson and M.W. Spong. Bilateral control of teleoperators with time delay. IEEE Trans. Autom. Control, 34(5):494-501, May 1989.

[3] G. Niemeyer and J.J. E. Slotine. Stable adaptive teleoperation. IEEE Journal of Oceanic Engineering, 16(1):152-162, Jan. 1991.

[4] S. Munir and W.J. Book. Internet-based teleoperation using wave variables with prediction. IEEE/ASME Trans. Mechatronics, 7(2):124133, June 2002.

[5] G. Niemeyer and J.J. E. Slotine. Towards force-reflecting teleoperation over the internet. Proc. of the IEEE Int. Conf. on Robot. and Autom., pages 1909-1915, May 1998.

[6] N.A. Tanner and G. Niemeyer. Practical limitations of wave controllers in teleoperation. Proc. of the IEEE Conf. on Robotics, Automation and Mechatronics, pages 25-30, December 2004.

[7] M. Alise, R.G. Roberts, and D.W. Repperger. The wave variable method for multiple degree of freedom teleoperation systems with time delay. Proc. of the IEEE Int. Conf. on Robot. and Autom., pages 2908-2913, May 2006.

[8] D. Lee and P.Y. Li. Passive bilateral control ant tool dynamics rendering for nonlinear mechanical teleoperators. IEEE Trans. Robot., 21(5):936-951, Oct. 2005.

[9] J.H. Ryu, C. Preusche, B. Hannaford, and G. Hirzinger. Time domain passivity control with reference energy following. IEEE Trans. Sys. Technology, 13(5):737-142, Sept. 2005.

[10] M. Mahvash and V. Hayward. High-fidelity passive force-reflecting virtual environments. IEEE trans. Robot., 21(1):38-46, Feb. 2006.

[11] D. Lee and M.W. Spong. Passive bilateral teleoperation with constant time delay. IEEE trans. Robot., 22(2):269-281, April 2006.

[12] N. Chopra, M.W. Spong, R. Ortega, and N. Barabanov. On tracking preformance in bilateral teleoperation. IEEE trans. Robot., 22(4):844847, Aug. 2006.

[13] G. Niemeyer and J.J. E. Slotine. Telemanipulation with time delays. Int. Jour. Robot. Research, 23(9):873-890, Sept. 2004.

[14] R. Ortega, N. Chopra, and M.W. Spong. A new passivity formulation for bilateral teleoperation with time delays. Proc. of the CNRS-NSF Workshop: Advances in time-delay systems, La Defense, Paris, Jan. 2003.

[15] N. Turro, O. Khatib, and E. Coste-Maniere. Haptically augmented teleoperation. Proc. of the IEEE Int. Conf. on Robot. and Autom., pages 386-392, May 2001.

[16] A. Casavola and M. Sorbara. Towards constrained teleoperation for safe long-distance robotic surgical operations. Proc. of the IEEE Int. Conf. on Robot. and Autom., pages 697-702, April 2005.

[17] C. Bohn and D.P. Atherton. An analysis package comparing pid antiwindup strategies. IEEE Control Syst. Mag., 15(2):34-40, April 1995. 